\title{
Rash in Adult and Pediatric Patients Treated with Lamotrigine
}

\author{
John A. Messenheimer
}

\begin{abstract}
Data from clinical trials with lamotrigine indicate that the risk of serious rash in pediatric patients is higher than in adults. The incidence of rash associated with hospitalization among adults treated with lamotrigine is $0.3 \%$ and among pediatric patients $1.0 \%$. The incidence of cases reported as possible Stevens-Johnson syndrome is $0.1 \%$ for adult patients and $0.5 \%$ for pediatric patients. These rates reflect lamotrigine dosing and concomitant AEDs used in these trials, both of which are risk factors for rash. Since many of the trials were conducted prior to the establishment of the current dosing recommendations, the incidence of serious rash in clinical trials does not necessarily reflect the incidence to be expected with use of current dosing recommendations. The higher incidence of serious rash in pediatric patients may at least partially be accounted for by the differential effects of the risk factors of dosing and concomitant use of valproic acid in these patients.
\end{abstract}

\begin{abstract}
RÉSUMÉ: Éruption cutanée chez les adultes et les enfants traités par la lamotrigine. Les données d'études cliniques sur la lamotrigine indiquent que le risque d'une éruption cutanée sévère sous lamotrigine chez les patients pédiatriques est plus élevé que chez les adultes. L'incidence d'une éruption cutanée associée à une hospitalisation chez les adultes traités par la lamotrigine est de $0.3 \%$ et chez les enfants de $0.9 \%$. L'incidence des cas rapportés comme présentant un SSJ possible est de $0.1 \%$ chez les adultes et de $0.5 \%$ chez les enfants. Ces taux reflètent la posologie de la lamotrigine et l'utilisation concomitante de médicaments antiépileptiques dans ces essais thérapeutiques, toutes deux étant des facteurs de risque d'une éruption cutanée. Comme plusieurs des essais thérapeutiques ont été faits avant l'établissement des recommendations actuelles sur la posologie, l'incidence d'éruptions cutanées sévères dans les essais thérapeutiques ne reflète pas nécessairement l'incidence à laquelle on doit s'attendre avec l'utilisation des recommendations courantes sur la posologie. L'incidence plus élevée d'une éruption cutanée sévère chez les enfants peut être expliquée, du moins en partie, par des effets différentiels des facteurs de risque de la posologie et de l'utilisation concomitante d'AVP chez ces patients.
\end{abstract}

Can. J. Neurol. Sci. 1998; 25: S14-S18

Evidence from multiple clinical trials, including over 3,000 adult patients and over 1,000 pediatric patients aged $<16$ years, indicates that lamotrigine is an effective, broad spectrum and well-tolerated antiepileptic drug (AED) in adult and pediatric patients. Adverse events have been infrequent and typically resolve promptly upon dose adjustment of lamotrigine or concomitant medications. The adverse event of greatest concern, and the one most often leading to the discontinuation of lamotrigine, has been rash. The estimates of the incidence of serious and non-serious rash with lamotrigine in clinical trials and postmarketing studies will be reviewed. The overall incidence of serious rash in pediatric patients is higher than in adults, possibly attributable to over-representation of concomitant valproic acid (VPA) and exceeding lamotrigine dosing recommendations in the pediatric group.

\section{Clinical Spectrum of Rash with Lamotrigine}

As with several other AEDs, a variety of rashes has been reported with the use of lamotrigine. These include simple morbillform rash, urticaria, angioedema, erythema multiforme, Stevens-Johnson syndrome (SJS), toxic epidermal necrolysis (TEN), and hypersensitivity syndrome (HSS). Rashes typically occur within the first eight weeks of treatment with only rare and questionable exceptions. Because of problems with the accuracy of dermatological diagnoses, this report will define as serious rash any rash that was associated with hospitalization or reported as possible SJS. This definition of serious rash is a conservative one: some of the cases reported as SJS would not be classified as such according to recently developed international diagnostic criteria. ${ }^{1}$ In addition, some patients hospitalized for a combination of events (e.g., increased seizures, rash, vomiting), may not have been hospitalized solely due to the seriousness of the rash.

\section{Stevens-Johnson syndrome (SJS) and toxic epidermal necrolysis (TEN)}

Stevens-Johnson syndrome (SJS) and toxic epidermal necrolysis (TEN) are related, severe, mucocutaneous disorders that are associated with any one of over a hundred possible medications, most commonly sulfonamides and AEDs. ${ }^{2}$ Over $80 \%$ of TEN and approximately $50 \%$ of SJS cases are drug related. ${ }^{2}$ The lower association of SJS with drug use may be due to frequent diagnostic confusion between SJS and erythema multiforme (EM). Erythema multiforme, which may also involve mucous

From Neurology and Psychiatry Clinical Development, Glaxo Wellcome, Inc. RECEIVED JULY 27, 1998.

Reprint requests to; John Messenheimer, Five Moore Drive, PO Box 13398, Research Triangle Park, NC USA 27709 
membranes, is most often seen in association with infections and can be difficult for the non-dermatologist to distinguish from SJS on clinical grounds alone. ${ }^{3}$ The estimated mortality rate for SJS is < 5\% while that for TEN is approximately $30 \%$. In a review of 87 cases of TEN Revuz et al. ${ }^{4}$ observed a mortality rate of $25 \%$ usually due to infection with Staphylococcus or Pseudomonas. Cutaneous pigmentary changes and sicca syndrome were frequently seen among survivors.

The mechanisms underlying these reactions are unknown. The pathological changes are those of a cell-mediated cytotoxic reaction directed against epidermal cells. ${ }^{2}$ One possible mechanism is the formation of reactive metabolites that covalently bind to protein and provide an antigenic stimulus for an immune response. A direct toxic effect of a reactive metabolite is also possible although the rapidity with which rechallenge precipitates a second reaction clearly indicates an immune process is involved.

A number of factors may increase the risk of SJS or TEN. Many patients who develop these conditions have underlying diseases. Infection and conditions that alter immunologic function are two factors that have been shown to increase the risk. For example, patients with AIDS have a $10-100$ fold increased risk of these reactions. ${ }^{2}$ Immunosuppressed organ transplant patients have developed TEN. The HLA phenotype B12 has been associated with an increased risk as well. ${ }^{2}$

\section{Anticonvulsant hypersensitivity syndrome (HSS)}

Despite severe skin involvement, most patients with SJS or TEN do not have significant evidence of involvement of other organ systems. However, a reaction termed the hypersensitivity syndrome (HSS) that includes rash and fever as well as a variable extent of involvement of other organ systems, is also associated with many different medications and especially AEDs (anticonvulsant hypersensitivity syndrome). ${ }^{5}$ Like SJS, these reactions also occur within the first few weeks of treatment. The mechanism underlying these reactions is unknown but may be related to the direct toxic effects of an active metabolite. The rash associated with this condition is typically morbilliform and not severe while the involvement of various other organ systems may be severe and, in rare cases, fatal.

The anticonvulsant hypersensitivity syndrome and SJS therefore appear to have different, although not mutually exclusive, pathophysiological mechanisms. It remains possible that they are both part of a single spectrum of drug-related reactions. Most patients with SJS however, despite the very severe involvement of skin, do not have significant hepatic or hematological abnormalities and most patients with HSS, who may have very severe involvement of one or more organ systems, do not have the severe cutaneous involvement seen with SJS.

\section{Diagnostic issues with cutaneous reactions to drugs}

The occurrence of rash in the setting of initiation of treatment with a medication can be a diagnostic challenge. The serious and non-serious forms of rash typically occur within the same time period in relation to the initiation of treatment. Severe forms of rash such as TEN that are characterized by significant amounts of epidermal loss are less of a diagnostic problem than SJS which can be confused with erythema multiforme. Viral exanthems commonly occur in children and can also be confused with milder forms of these more severe conditions.
The initial manifestations of the HSS may include fever and pharyngitis in the absence of rash, leading to the introduction of antipyretics and antibiotics which are also associated with the development of severe cutaneous reactions. Probably the most frequent area of diagnostic confusion is between erythema multiforme (EM) and SJS. It has recently been shown ${ }^{3}$ that EM with mucous membrane involvement and SJS, which are difficult for non-dermatologists to distinguish on clinical grounds, are actually different disorders clinically and have very different etiologies. Erythema multiforme is most often associated with herpes infections or other non-drug related causes while SJS is not related to herpes infection and is almost always drug related.

In addition, the background rates of serious rash appear to be increased in younger patients. In one epidemiological study utilizing Group Health Cooperative (GHC) discharge diagnoses over a 14 year period, the risk of hospitalization for SJS, TEN or erythema multiforme was found to be substantially higher in patients $<20$ years of age (7 per million person-years) than for people aged 20 - 64 years (1.8 per million person-years) ${ }^{6}$ In this report the incidence of erythema multiforme, SJS, or TEN was highest ( 35 per million person-years) for those under the age of 5 where approximately $30 \%$ of cases were found to be not drug-related.

This report reviews all sources of data that are sufficient to permit reasonable estimates of the incidence of cutaneous reactions to lamotrigine. In this context, HSS leading to multiorgan failure will not be discussed as this reaction has not been reported in any of these data sources. There have been spontaneous post marketing reports of multiorgan failure with the use of lamotrigine, many of which have been attributable to sepsis or status epilepticus. However, multiorgan failure with lamotrigine use has also been reported as a manifestation of hypersensitivity to lamotrigine. ${ }^{7}$ The absence of cases of multiorgan failure from the clinical trial database and other sources of data is an indication that the low incidence of these events. The estimated incidence of multiorgan failure related to HSS with carbamazepine, phenytoin or phenobarbital is $1 / 10,000 .^{5}$

\section{Clinical trial data}

The clinical trial database for lamotrigine was examined for all cases of rash using a data cut-off of December 1996 and including only those patients for whom complete information was available regarding the dosing of lamotrigine and concomitant AEDs. This analysis was undertaken to investigate the effect of the risk factors of lamotrigine dosing and concomitant AEDs on the incidence of rash.

Table 1 lists the incidence of all cutaneous events in lamotrigine pediatric and adult clinical trials. The rates of all rash (10.7\% adult, $12.9 \%$ pediatric) and rash leading to discontinuation (3.5\% adults, $4.8 \%$ pediatric) are not statistically significantly different for adult and pediatric patients. Serious rash, defined as rash leading to hospitalization or rash reported as SJS, occurred in $1.0 \%$ of pediatric patients compared with $0.3 \%$ of adult patients.

\section{Post-marketing studies}

A prospective observational study (ALERT Study) to measure the risk of serious adverse events in patients 16 and older and initiating lamotrigine therapy was conducted in the U.S.A. Of 878 patients enrolled through practicing neurologists, 4 month follow-up forms were received for 767 patients. One 
Table 1:. Incidence of rash events in adult and pediatric clinical trials with lamotrigine.

\begin{tabular}{lr}
\hline ADULT (> 16 YEARS) & TOTAL (\%) \\
\hline $\mathrm{N}$ & 3348 \\
\hline RASH & $359(10.7 \%)$ \\
\hline RASH LEADING TO DISCONTINUATION & $118(3.5 \%)$ \\
\hline RASH ASSOCIATED WITH HOSPITALIZATION & $11(0.3 \%)$ \\
\hline POSSIBLE SJS & $4(0.1 \%)$ \\
\hline SJS OR HOSPITALIZATION & $11(0.3 \%)$ \\
\hline PEDIATRIC (< 16 YEARS) & 1071 \\
\hline N & $138(12.9 \%)$ \\
\hline RASH & $51(4.8 \%)$ \\
\hline RASH LEADING TO DISCONTINUATION & $10(0.9 \%)$ \\
\hline RASH ASSOCIATED WITH HOSPITALIZATION & $5(0.5 \%)$ \\
\hline POSSIBLE SJS & $11(1.0 \%)$ \\
\hline SJS OR HOSPITALIZATION & \\
\hline As one pediatric patient with possible SJS was not hospitalized the \\
incidence of “serious rash" in pediatric clinical trial patients is \\
ll/l071 (1.0\%).
\end{tabular}

patient was reported to have Stevens-Johnson syndrome and was hospitalized; and one was reported as a hypersensitivity rash also involving hospitalization. Therefore, the risk of serious rash was 2.6 per 1000 or $0.26 \%$.

\section{Epidemiologic studies of SJS/TEN with AED use including lamotrigine}

\section{Lamotrigine Prescription Event Monitoring (PEM)}

To measure the safety profile in a large population of lamotrigine users, an observational study of lamotrigine users was conducted by the Drug Safety Research Unit (DSRU) at the University of Southampton, U.K. ${ }^{8}$ All first-time users of lamotrigine between December 1991 and February 1995 were identified through General Practitioner (GP) prescriptions from the national British Prescription Pricing Authority. Six months after the first lamotrigine prescription, a follow-up form was sent to each prescribing GP. In this form the GP listed any adverse event, regardless of cause, occurring since the first lamotrigine prescription. The DSRU followed up for more detailed information on any significant medical events, such as hospitalization for rash and reported SJS. Lamotrigine was not licensed for use in pediatric patients in the U.K. before May 1994, and pediatric dosing guidelines and the formulation (5 mg tablets) of lamotrigine needed to dose many children correctly were not available prior to licensing for use in pediatric patients.

Of 19,448 6-month green forms posted, follow-up data on 11,316 patients were collected. There were 12 events reported as Stevens-Johnson syndrome (SJS). Therefore, the overall risk of reported SJS was 1.1 per 1000 , and the risk of hospitalization related to rash was 1.1 per 1000 .

Within this study population, there were 1598 children and 9143 adults with known duration of lamotrigine exposure. Within the group of children 12 years or younger there were 5 cases of reported SJS, and one reported erythema multiforme involving hospitalization. The risk of serious rash in children aged 212 was therefore $6 / 1598$ or $0.38 \%$ and the risk of reported SJS was $5 / 1598$, or $0.31 \%$. The risk of reported SJS in children was substantially higher than the risk observed in subjects $>12$ years of age (7/9143 or $0.08 \%)$.

\section{Incidence of serious rash with other AEDs}

\section{Saskatchewan study}

Although serious and non-serious cutaneous reactions to a number of AEDs are well reported, accurate estimates of the incidence of these reactions have been limited. A recent study utilized anonymized data from the Saskatchewan Health Plan, a government insurance program which covers $>95 \%$ of the Saskatchewan population, to define an estimate of the incidence of serious cutaneous reactions among new users of phenytoin, carbamazepine or valproate. ${ }^{9}$ Based on an expert dermatologist's review of hospital discharge summaries, the incidence of rash associated with hospitalization was $8 / 8888,0.09 \%$ for new phenytoin users and $6 / 9,738,0.06 \%$ for new carbamazepine users. Although no cases of serious rash were reported among the 1504 valproate users in this study, serious rash related to the use of valproate was reported in the European case control study. ${ }^{1}$

\section{Rash with other AEDs in children}

Reports of the incidence of cutaneous reactions in children are more limited than reports in adults. As noted above, the background incidence of serious rash is higher in children than in adults. ${ }^{6}$ One retrospective study reported an incidence of $2 / 335,0.6 \%$ of SJS in pediatric patients treated with carbamazepine. ${ }^{10}$ In addition, sixteen patients $(4.8 \%)$ had evidence of hematological or hepatic dysfunction in association with rash suggesting the anticonvulsant hypersensitivity syndrome.

These studies cannot be used to directly compare with the lamotrigine studies but they do suggest that rash and serious rash is not a rare event with the use of other AEDs and that the incidence of these events may also be higher among children.

\section{Possible risk factors affecting the incidence of rash}

As dosing of lamotrigine (starting dose and rate of escalation) and the patterns of concomitant AED use, particularly VPA, are different for adult and pediatric patients, it is difficult to isolate the effect of these possible risk factor from age alone.

\section{Dosing as a risk factor for serious rash with lamotrigine}

There is evidence that dosing can influence the incidence of some reactions to phenytoin and carbamazepine. ${ }^{11,12}$ This also appears to be true for lamotrigine. Because of the low incidence of the more severe reactions, an effect of dosing on the incidence of SJS or severe HSS would be difficult to detect. There is, however, a clearly demonstrated relationship between dosing and the less severe forms of rash seen with these drugs. In 1978 Wilson et al. prospectively documented a high incidence of a concentration-dependent skin reaction in children treated with phenytoin. ${ }^{12}$ In this study five of ten children developed rash. All patients who had achieved a phenytoin serum concentration higher than 14 micro molar by the fifth day of treatment developed rash, while no rash occurred in patients with lower phenytoin concentrations. In 1984 Chadwick reported results 
from two prospective studies in which high initial serum concentrations were associated with an increased incidence of rash with phenytoin (6\%) and carbamazepine (16.6\%)." A relationship between dosing and various cutaneous manifestations of drug reactions has also been reported for several antibiotics and for captopril.

\section{Dosing as a risk factor for non-serious rash with lamotrigine in adults}

Figure 1 summarizes the relationship of lamotrigine starting dose to the incidence of discontinuation due to rash for all adult monotherapy studies. None of these patients developed serious rash.

These data clearly indicate that the incidence of rash leading to discontinuation of lamotrigine is strongly influenced by the starting dose of lamotrigine. Similar effects can be demonstrated for the rate of dose escalation over the first five weeks of treatment.

In the PEM study, detailed dosing information was not collected and an effect of dosing cannot be demonstrated. However, the fact that the $5 \mathrm{mg}$ pediatric dosing formulation of lamotrigine was not available on the market in the U.K. until the last year of the study suggests that dosing, particularly in those patients taking concomitant VPA, may have contributed to the increased incidence of rash in this study.

\section{Evidence that the rate of serious rash can be affected by dosing of lamotrigine}

The strongest evidence in support of an effect of dosing on the incidence of SJS/TEN with lamotrigine is the experience in Germany where a registry for serious cutaneous reactions been established since 1990 . This academically based registry, structured as an intensive reporting system, regularly contacted $100 \%$ of burn units, departments of pediatrics, departments of dermatology, and all internal medicine departments in hospitals with intensive care facilities or with more than 200 beds in western Germany and since 1996 in all of Germany. Thus, in

\section{Initial Dose and \% DC due to Rash}

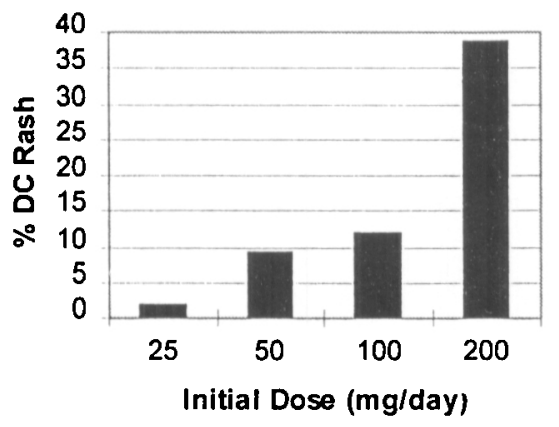

Figure 1: Initial lamotrigine dose and \% discontinuation of treatment due to rash. This graph illustrates the effect of initial dosing of lamotrigine on rash leading to discontinuation of treatment. The initial doses are shown on the $X$ axis. All of these data are from monotherapy trials in treatment-naïve adult subjects. All trials involved epilepsy patients except for the trial that initiated lamotrigine at $200 \mathrm{mg} /$ day which was a study in patients with migraine.
Germany almost all hospitalized cases of SJS and TEN are detected. Because of the prospective expert review of potential cases in this registry, these are confirmed cases of SJS or TEN. ${ }^{13}$ To ascertain retrospectively whether any cases have been missed, the registry regularly sends letters to all relevant hospital units. Lamotrigine was first marketed during the last 6 months of 1993 and the registry had been operating several years in advance of that. In 1993, five lamotrigine-related SJS or TEN cases were reported, four of whom were also taking valproic acid (M. Mockenhaupt, personal communication), and an estimated 1000-2500 patients were exposed. In the third quarter of 1993, Glaxo Wellcome amended the dosing regimen and initiated a campaign to inform physicians of the risks associated with excessively rapid lamotrigine dose escalations. The recommended starting dose of lamotrigine when used with valproic acid was reduced, from $50 \mathrm{mg}$ qd to $25 \mathrm{mg}$ qod. In each of the subsequent two years, two SJS or TEN cases were registered, and in 1996 no cases of SJS or TEN were reported on lamotrigine. During 1994, 1995, and 1996, sales of lamotrigine increased every year. The rate of SJS and TEN decreased with the implementation of the reduced dosing schedule, despite increased use of lamotrigine. These data are consistent with the hypothesis that reduced initial dosing and slower rates of dose escalation can reduce the rate of SJS associated with lamotrigine use. The German Registry focused on hospitalized cases only. Due to strict confidentiality laws in Germany, it is not feasible to review hospital records to determine if there were missed cases. However, record review is permitted for retrospectively ascertained cases. During the years 1990 - 1992, immediately prior to lamotrigine availability, the number of total SJS or TEN cases reported to the registry in each year and the estimated risk of all SJS or TEN in the general population increased in each year from 1.17 per million $(\mathrm{n}=75)$ to 1.89 per million $(\mathrm{n}=$ 125). ${ }^{13}$ This trend suggests that case ascertainment was steadily improving, rather than declining throughout the life of the registry. In addition, the registry has developed regular communication with $95 \%$ of all relevant units, suggesting that ascertainment is quite complete.

In summary, the data from adult patients clearly demonstrate an effect of dosing on the incidence of non-serious rash. There is circumstantial evidence to suggest that dosing may also be a factor influencing the incidence of serious rash.

\section{VPA as a risk factor}

Because of a wide range of dosing used in clinical trials, it is difficult to separate lamotrigine dosing and concomitant VPA as independent risk factors. The incidence of rash associated with hospitalization or possible SJS is higher when lamotrigine is combined with VPA in the absence of enzyme inducing AEDs than when lamotrigine is used without VPA or when lamotrigine is used with VPA in the presence of enzyme inducing AEDs (Table 2). Among 149 pediatric patients taking lamotrigine with VPA and enzyme inducers there were no cases of serious rash and no cases of rash leading to discontinuation. The PEM study also suggests an increased risk with the concomitant use of VPA and lamotrigine. In this study all five of the reported cases of SJS in pediatric patients were taking concomitant VPA. Although concomitant AED use in this study was not documented for all patients, a recent unpublished General Practice Research Database study showed that $41 \%$ of children $<12$ who 
Table 2: Rash rates with lamotrigine and various concomitant AED combinations

\begin{tabular}{|c|c|c|c|}
\hline $\begin{array}{l}\text { ADULT } \\
(>16 \text { YEARS) }\end{array}$ & $\begin{array}{l}\text { VPA ONLY } \\
\text { or } \\
\text { VPA+NEI }\end{array}$ & VPA+EI & NO VPA \\
\hline $\mathrm{N}$ & 205 & 301 & 2842 \\
\hline RASH & $40(19.5 \%)$ & $28(9.3 \%)$ & $291(10.2 \%)$ \\
\hline DC DUE TO RASH & $25(12.2 \%)$ & $10(3.3 \%)$ & $83(2.9 \%)$ \\
\hline HOSP DUE TO RASH & $3(1.5 \%)$ & $2(0.7 \%)$ & $5(0.2 \%)$ \\
\hline SJS & $1(0.5 \%)$ & $0(0 \%)$ & $3(0.1 \%)$ \\
\hline SIS OR HOSP & $3(1.5 \%)$ & $2(0.7 \%)$ & $5(0.2 \%)$ \\
\hline \multicolumn{4}{|l|}{$\begin{array}{l}\text { PEDIATRIC } \\
\text { (<16 YEARS) }\end{array}$} \\
\hline $\mathrm{N}$ & 294 & 149 & 628 \\
\hline RASH & $60(20.4 \%)$ & $7(4.7 \%)$ & $71(11.3 \%)$ \\
\hline $\begin{array}{l}\text { DC DUE } \\
\text { TO RASH }\end{array}$ & $28(9.5 \%)$ & $0(0 \%)$ & $23(3.7 \%)$ \\
\hline $\begin{array}{l}\text { HOSP DUE } \\
\text { TO RASH }\end{array}$ & $4(1.4 \%)$ & $0(0 \%)$ & $6(1.0 \%)$ \\
\hline SJS & $4(1.4 \%)$ & $0(0 \%)$ & $2(0.3 \%)$ \\
\hline SJS OR HOSP & $5(1.7 \%)$ & $0(0 \%)$ & $6(1.0 \%)$ \\
\hline $\begin{array}{l}\text { EI = Enzyme-inducing } \\
\text { NEI = Non-enzyme indu } \\
\text { DC = Discontinuation of } \\
\text { HOSP = Hospitalization } \\
\text { VPA = Valproic acid } \\
\text { SJS = Stevens-Johnson s }\end{array}$ & $\begin{array}{l}\text { D } \\
\text { ng AED } \\
\text { amotrigine }\end{array}$ & & \\
\hline
\end{tabular}

received a lamotrigine prescription were also taking VPA. If this rate of concomitant VPA is assumed to be correct for the PEM database, the risk of SJS among children taking lamotrigine with concomitant VPA would be $5 / 655,0.8 \%$ while the rate among children not taking concomitant VPA would be $0 / 942$. The mechanism by which valproic acid increases the risk of rash with lamotrigine is not known. As dosing of lamotrigine is also a risk factor and valproic acid inhibits the metabolism of lamotrigine it is possible that the mechanism is pharmacokinetic rather than metabolic.

\section{Management of rash}

Although adherence to the dosing recommendations of lamotrigine will not eliminate the risk of rash, it is essential to mini- mize the risk associated with rash by adherence to manufacturer's dosing recommendations. The likelihood of a serious rash developing from a mild rash is unknown. However, in the event of rash occurring within the first few months of treatment, lamotrigine should be discontinued unless the rash is clearly not drug-related. Patients developing a rash should be seen as quickly as possible by a health professional familiar with rash diagnosis. Cases with serious rash may require hospitalization.

\section{REFERENCES}

1. Roujeau JC, Kelly JP, Naldi L, et al. Medication use and the risk of Stevens-Johnson syndrome or toxic epidermal necrolysis. N Engl J Med 1995; 333: 1600-1607.

2. Roujeau JC, Stern RS. Severe adverse cutaneous reactions to drugs. N Engl J Med 1994; 331: 1272-1285.

3. Assier H, Bastuji-Garin S, Revuz J, Roujeau JC. Erythema multiforme with mucous membrane involvement and Stevens-Johnson syndrome are clinically different disorders with distinct causes. Arch Dermatol 1995; 131: 539-543.

4. Revuz J, Penso D, Roujeau JC, et al, Toxic epidermal necrolysis. Clinical findings and prognosis factors in 87 patients. Arch Dermatol 1995; 123: 1160-1165.

5. Shear NH, Spielberg SP. Anticonvulsant hypersensitivity syndrome. An in-vitro assessment of risk. J Clinical Invest 1988; 82: 18261832.

6. Chan HL, Stern RS, Arndt K, et al. The incidence of erythema multiforme, Stevens-Johnson syndrome, and toxic epidermal necrolysis. Arch Dermatology 1990; 126: 43-47.

7. Chattergoon DS, McGuigan MA, Koren G, Hwang P, Ito S. Multiorgan dysfunction and disseminated intravascular coagulation in children receiving lamotrigine and valproic acid. Neurology 1997; 19: 1442-1444.

8. Mackay FJ, Wilton LV, Pearce GL, Freemantle SN, Mann RD. Safety of long-term lamotrigine in epilepsy. Epilepsia 1997; 38: 881886.

9. Tennis P, Stern R. A Saskatchewan record linkage study on the risk of hospitalizations for serious cutaneous disorders occurring after initiation of use of phenytoin, carbamazepine, and sodium valproate. Neurology 1997; 49: 1-5.

10. Konishi T, Naganuma Y, Hongo K, et al. Carbamazepine-induced skin rash in children with epilepsy. Euro J Pediatrics 1993; 152: 605-608.

11. Chadwick D, Shaw MDM, Foy P, Rawlins MD, Turnbull DM. Serum anticonvulsant concentrations and the risk of drug induced skin eruptions. J Neurol Neurosurg Psychiatry 1984; 47: 642-644.

12. Wilson JT, Hojer B, Tomson G, Rane A, Sjoqvist F. High incidence of a concentration dependent skin reaction in children treated with phenytoin. Br Med J 1978; 1(6127): 1583-1586.

13. Rzany B, Mockenhaupt M, Baur S, et al. Epidemiology of erythema exsudativum multiforme majus, Stevens-Johnson syndrome, and toxic epidermal necrolysis in Germany (1990-1992): structure and results of a population-based registry. J Clin Epidemiol 1996; 49: 769-773. 